Article

\title{
Dermal Targeting Delivery of Terbinafine Hydrochloride Using Novel Multi-Ethosomes: A New Approach to Fungal Infection Treatment
}

\author{
Lijun Zhang ${ }^{1,+}{ }^{+}$Xue $\mathrm{Li}^{2,{ }^{\dagger}}$, Shunyao Zhu ${ }^{1}$, Ting Zhang ${ }^{1}$, Aikebaier Maimaiti ${ }^{1}$, Meihong Ding ${ }^{1}$ \\ and Senlin Shi ${ }^{1, * \mathbb{D}}$ \\ 1 College of Pharmaceutical Science, Zhejiang Chinese Medical University, Hangzhou 310053, China; \\ lijunzhang@zcmu.edu.cn (L.Z.); zsy@zcmu.edu.cn (S.Z.); zhangting55@163.com (T.Z.); \\ akpar15@163.com (A.M.); dingmeihong0125@163.com (M.D.) \\ 2 Institut des Sciences Moléculaires d'Orsay, CNRS, Université Paris-Saclay, 91405 Orsay, France; \\ xue.li@universite-paris-saclay.fr \\ * Correspondence: pjstone@zcmu.edu.cn; Tel.: +86-571-61768135 \\ + These authors contributed equally to this work.
}

Received: 25 February 2020; Accepted: 23 March 2020; Published: 25 March 2020

\begin{abstract}
This research aimed to develop and evaluate a novel multi-ethosome (ME) system for the dermal delivery of terbinafine hydrochloride (TH) as a new approach to fungal infection treatment. TH-loaded MEs were successfully prepared using cinnamaldehyde as a penetration enhancer. Mean diameter of ME was found as $\sim 100 \mathrm{~nm}$ with monodispersed size distribution. Drug entrapment efficiency reached up to $86 \% \pm 1.4 \%$. MEs exhibited excellent colloid stability and no drug leakage after 2 months of storage. In contrast to a commercial Lamisil ${ }^{\circledR}$ cream, ME significantly improved the targeting efficiency by increasing the fluidity of stratum corneum layer, revealed by attenuated total reflection Fourier transformed infrared spectroscopy (ATR-FTIR). The dermal targeting effect was visualized using confocal microscopy. Moreover, skin irritation and allergy tests showed that ME was not irritating to the skin. The improved antifungal activity of ME was proved in vitro on Candida albicans strains by minimal inhibitory concentration (MIC) assay. This study paves the way towards design of MEs for dermal fungal infection treatment.
\end{abstract}

Keywords: multi-ethosomes; cinnamaldehyde; terbinafine hydrochloride; targeting delivery; antifungal

\section{Introduction}

Terbinafine hydrochloride $(\mathrm{TH})$ is a synthetic allylamine derivative with antifungal activity, mainly used to treat topically superficial skin infections, such as tinea corporis, tinea pedis, and Candida species infections [1]. Generally, when administrated orally, TH causes systemic side effects, including liver toxicity and gastrointestinal complications [2]. TH creams and gels are developed clinically for superficial skin infections, because TH accumulates mainly in superficial skin and adipose tissue [3] due to its high lipophilicity. Unfortunately, it is poorly permeable across the stratum corneum (SC) to reach the lesion [4], and therefore it lacks efficacy in dermal fungal infections. Clinically, it is required to increase the administered doses (e.g., three times a day for more than 6 weeks), which usually lead to side effects (such as skin keratinization) and drug resistance. Therefore, the development of a targeting drug delivery system to improve the drug efficiency is urgently required, which would reduce the side effects. This study aimed to develop and evaluate a novel nanoparticulate system for dermal delivery of $\mathrm{TH}$ as a new approach to fungal infection treatment.

Nanotechnology has revolutionized drug delivery in particular by achieving drug targeting and transcytosis of drugs across biological barriers. It has been reported that some nanoparticles like 
liposomes enable effective delivery of encapsulated molecules to target lesion sites, thus minimizing systemic toxicity [5]. More recently, ethosome, a modification of liposomes by addition of ethanol, attracts increasing interest in transdermal administration. As shown in Figure 1, classical ethosome (CE) contains ethanol in both the aqueous inner phase and the lipid bilayer, leading to the deformability of the formulation. Advantageously, CE showed better skin permeation compared with liposomes [6]. This is mainly due to the ethanol it contains, which could interfere with the orderly SC arrangement, contributing to better drug penetration across the SC barrier. Binary ethosome (BE) further enhances these properties by addition of other alcohols (such as propylene glycol (PG)), which is favorable to increase the drug solubility, improve the drug entrapment efficiency and stability of the formulation [7]. On the other hand, transethosome (TE) contains edge activator (e.g., surfactants) and/or penetration enhancers [8] to increase the drug penetration by modifying the drug thermodynamic activity, and helping to solubilize it in the SC lipids. These advantages allow ethosome to find useful applications in the dermal targeting drug delivery system [9]. However, there are still rare of examples used clinically [10] due to the low yield, and skin irritation or dermatitis. In this study, we propose a multi-ethosome (ME) combining the advantage of both BE and TE. Recently, a novel nanovesicle "Flexosome" was described for enhanced transdermal drug delivery where different edge activator combinations (Tween 80, Span 85, hexadecyl trimethyl ammonium bromide, sodium deoxycholate, etc.) were used. Cholesterol was added in the phospholipids to improve the rigidity and stability of the formulation [11]. According to its composition, this flexosome is actually a specific type of TE. To the best of our knowledge, we report here for the first time a ME composed of phospholipids, ethanol, PG, edge activator (Tween 80), and a penetration enhancer (cinnamaldehyde (CA)). CA is a natural penetration enhancer obtained from cinnamon, and interestingly, it possesses antifungal property [12]. We bring the proof of concept that the ME has expected advantages over the BE and TE.

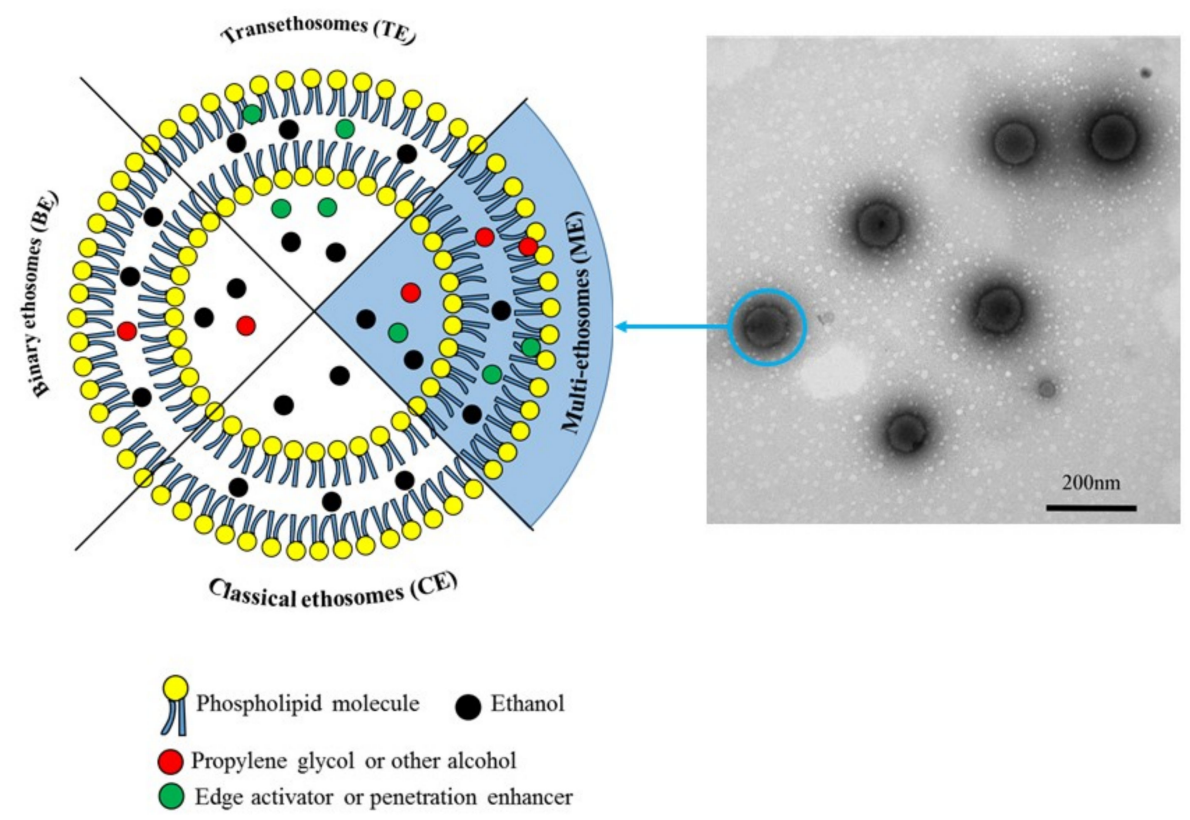

Figure 1. Schematic representation of ethosomes and transmission electron microscopy (TEM) images of multi-ethosomes.

In this study, TH-loaded MEs with homogeneous sizes of $\sim 100 \mathrm{~nm}$ were successfully prepared via a cold method. High drug entrapment efficiency (EE) reached up to $86 \%$. MEs possessed excellent colloid stability with no drug leakage after 2 months storage. In contrast to a commercial Lamisil ${ }^{\circledR}$ cream, ME exhibited the targeting property by increasing the fluidity of SC layer. Rhodamine-labeled MEs were successfully prepared for confocal microscopy to visualize ME penetration across the SC layer. 
Moreover, ME was found biocompatible, which was not irritating to the skin, confirmed by irritation and allergy tests. Finally, the antifungal activity of ME was tested in vitro on Candida albicans strains.

\section{Materials and Methods}

\subsection{Materials}

\subsubsection{Chemicals}

TH (99.9\%) and CA were kindly provided from National Institutes for Food and Drug Control (Beijing, China). Egg Yolk Lecithin (PEL, PL-100M) was obtained from Shanghai Advanced Vehicle Technology Pharmaceutical L.T.D. (Shanghai, China). Ethanol (Roswell Park Memorial Institute-1640 (RPMI-1640)), PG, phosphate buffer saline (PBS), and Tween 80 were purchased from Fisher Scientific (Loughborough, UK). Sabouraud Dextrose Agar and Sabouraud Dextrose Broth were received from Hopebio (Qingdao, China). Rhodamine B (Rh B) and Sephadex ${ }^{\circledR}$ G-50 were supplied by Sigma Aldrich (Steinheim, Germany). Lamisil ${ }^{\circledR} 1 \%$ cream was purchased from Novartis (Shanghai, China). All the chemicals were of analytical grade.

\subsubsection{Animals}

Male Sprague-Dawley (SD) rats $(200 \pm 20 \mathrm{~g})$ and rabbits $(2.5 \pm 0.5 \mathrm{~kg})$ were obtained from the Laboratory Animal Research Center of Zhejiang Chinese Medical University. All of the animals were housed in animal facilities at $25 \pm 2{ }^{\circ} \mathrm{C}$ and relative humidity $50 \% \pm 2 \%$, under a 12:12 h light and dark cycle. Food and water are allowed ad libitum for rats and rabbits.

\subsection{TH Solubility Determination}

The solubility of TH in different surfactant mediums was evaluated to satisfy the sink conditions [13]. Briefly, excessive $\mathrm{TH}$ was added to $10 \mathrm{~mL}$ of different medium at $37.0 \pm 0.5^{\circ} \mathrm{C}$, the suspensions were under vigorously shaking for $48 \mathrm{~h}$. The suspension was centrifuged at $1500 \times g$ and the pellet was discarded. The TH content in the supernatant was measured by an UV spectrophotometer at $283 \mathrm{~nm}$. The experiments were performed in triplicate.

\subsection{Preparation of Vesiclular Systems}

Three different ME formulations loaded with TH (detailed in Table 1, ME-1, 2, and 3) were prepared by adjusting a cold method to that of Touitou et al. [14]. Briefly, TH $(0.5 \%, w / v)$, PEL (2\%, $w / v)$, Tween $80(5 \%, w / v)$, and CA (in the range of $0.1 \%$ to $0.5 \%, w / v$ ) were dissolved in $32.5 \mathrm{~mL}$ of ethanol and PG mixture $(7: 3, v / v)$ in a closed vessel by stirring at $850 \mathrm{rpm}$. Then, $67.5 \mathrm{~mL}$ of PBS ( $\mathrm{pH}$ 7.8) was slowly added to the vessel by peristaltic pump and maintained at $30 \pm 1{ }^{\circ} \mathrm{C}$ for $15 \mathrm{~min}$. Eventually, it was extruded through a membrane filter $(100 \mathrm{~nm}$, Minisart, Sartorius Stedim, Gottingen, Germany) to obtain ME formulations.

Table 1. Composition of the different ethosome formulations and their physico-chemical properties, as well as the drug entrapment efficiency.

\begin{tabular}{ccccccc}
\hline Formulation & $\begin{array}{c}\mathbf{C A} \\
(\mathbf{\%}, \boldsymbol{w} / \boldsymbol{v})\end{array}$ & $\begin{array}{c}\mathbf{P G}^{\mathbf{a}} \\
(\mathbf{\%}, \boldsymbol{v} / \boldsymbol{v})\end{array}$ & Size $(\mathbf{n m})$ & PDI & Zeta (mv) & EE (\%) \\
\hline ME-1 & 0.1 & 30 & $120 \pm 8.2$ & $0.28 \pm 0.08$ & $-7.01 \pm 1.29$ & $77.6 \pm 3.0$ \\
ME-2 & 0.3 & 30 & $103 \pm 9.3$ & $0.22 \pm 0.05$ & $-11.95 \pm 1.42$ & $86.4 \pm 1.7$ \\
ME-3 & 0.5 & 30 & $100 \pm 8.9$ & $0.21 \pm 0.03$ & $-13.02 \pm 1.21$ & $76.3 \pm 1.8$ \\
BE & 0 & 30 & $132 \pm 9.7$ & $0.30 \pm 0.05$ & $-5.40 \pm 1.83$ & $65.9 \pm 1.9$ \\
TE & 0.3 & 0 & $124 \pm 6.9$ & $0.28 \pm 0.06$ & $-10.11 \pm 1.29$ & $78.9 \pm 2.4$ \\
CE & 0 & 0 & $152 \pm 7.2$ & $0.30 \pm 0.04$ & $-10.91 \pm 2.63$ & $60.9 \pm 1.9$ \\
\hline
\end{tabular}

Note: a: the percentage of PG in ethanol phase, which corresponding to ethanol and PG mixture $(7: 3, v / v)$. Data are shown as the mean $\pm \mathrm{SD}(n=3)$. 
To study the effect of CA and PG, TH-loaded binary ethosome (BE) and TH-loaded transethosome (TE) were prepared as listed in Table 1. As control, TH-loaded classical ethosome formulation (CE) was prepared in the same procedure except that neither PG nor CA were added. All the formulations were stored at $4{ }^{\circ} \mathrm{C}$ environment for further investigation.

$\mathrm{Rh}$ B-labeled formulations were prepared for visualization by adding $50 \mathrm{mg}$ of $\mathrm{Rh} \mathrm{B}$ in the ethanol phase using the same protocol as described.

\subsection{Characterization of Formulations}

\subsubsection{Hydrodynamic Diameter and Zeta Potential}

The hydrodynamic diameter, polydispersity index (PDI), and zeta potential (zeta) of prepared vesicular formulations were assessed using a Nano-ZetaSizer (ZS90, Malvern, UK) with dynamic and electrophoretic light scattering [15]. Measurements were performed in triplicate from three independent samples at $25^{\circ} \mathrm{C}$.

\subsubsection{Transmission Electron Microscopy (TEM)}

The morphology of the formulation was characterized by TEM. The samples were added onto a carbon film and negatively stained by phosphomolybdic acid, then dried at room temperature for $1 \mathrm{~h}$ [16]. Images were captured by H7650 transmission electron microscope (HITACHI, Tokyo, Japan) at an acceleration voltage of $80 \mathrm{kV}$.

\subsubsection{Drug Entrapment Efficiency (EE)}

The $\mathrm{EE}$ of the ME formulas were determined by separating the unencapsulated drug from vesicles by microcolumn centrifugation [17]. Briefly, TH-loaded formulations were evenly added to the columns (Sephadex ${ }^{\circledR}$ G-50), and then centrifuged at $1600 \times g$ for $3 \mathrm{~min}$. After elution and centrifugation for three times, the clear supernatant was collected and ruptured with methanol to recover the unencapsulated drug. Finally, TH amount was quantified by recording the absorbance at $283 \mathrm{~nm}$. The EE\% was calculated as Equation (1):

$$
\mathrm{EE} \%=W_{\text {ent }} / W_{\text {tol }} \times 100 \%
$$

where $W_{t o l}$ is the total amount of TH and $W_{\text {ent }}$ is the amount of TH entrapped in the formulations.

\subsubsection{Physical Stability Studies}

Stability studies of the developed formulations were performed at temperature of $4 \pm 2$ and $25 \pm 2{ }^{\circ} \mathrm{C}$ stored in brown glass bottles. The hydrodynamic diameter and $\mathrm{EE} \%$ were measured after 60 days storage [18].

\subsection{Ex Vivo Drug Permeation and Deposition Studies}

\subsubsection{Franz Diffusion Cell Methods}

For ex vivo drug permeation and deposition studies, the abdominal hair of SD male albino rats was shaved using an electric razor after sacrificing with excess isoflurane inhalation. The abdominal skin was surgically removed. The adhered subcutaneous tissue or fat was carefully separated with a scalpel. The integrity of skin samples was carefully checked before usage to ensure that the skin samples were suitable for subsequent experiments. Ex vivo drug permeation study was performed with the TH-loaded formulations (CE, BE, TE, and ME-2) using a Franz glass diffusion cell (SFDC 6, LOGAN, Somerset, NJ, USA). The effective permeation area of the diffusion cell and receptor cell volume were $1.13 \mathrm{~cm}^{2}$ and $5 \mathrm{~mL}$, respectively. The diffusion cell was covered with a black nylon membrane to avoid evaporation and protect from light. The skin sample was mounted between the donor and receptor compartment with the SC side towards the donor chamber [19]. The receiving 
solution was PBS ( $\mathrm{pH}=7.4)$ with the addition of Tween $80(0.01 \%, w / v)$, which was maintained at $37.0 \pm 1{ }^{\circ} \mathrm{C}$ to meet the sink conditions (Figure S1). Equivalent formulations containing $5 \mathrm{mg}$ TH were applied to the surface of the rat skin. Market product of TH (Lamisil ${ }^{\circledR} 1 \%$ cream) was used as control.

After 6, 12, and $24 \mathrm{~h}$, the skin was removed from Franz diffusion cell and washed with water. The surface was dried with filter paper, and different skin layers were separated by a tape stripping method [20]. All strips (deposited in SC) and skin tissue (deposited in epidermis and dermis) were collected and kept in a centrifuge tube containing $10 \mathrm{~mL}$ of methanol as drug extraction solvent, followed by sonication in an ice bath for $4 \mathrm{~min}$ and shaking in the dark for $12 \mathrm{~h}$ [21]. The tissue suspension was finally centrifuged at $1500 \times g$. The supernatant was collected and further filtered by microporous filter $(0.45 \mu \mathrm{m})$. The content of TH in different skin layers was quantified by UV-Visible Spectrophotometer. Some related transdermal penetration parameters was calculated. Targeting efficiency was calculated to evaluate the targeting effect of the formulations.

Targeting efficiency was calculated as Equation (2):

$$
\begin{gathered}
\text { Targeting efficiency = total amount of TH deposited in skin with } \\
\text { formulations/total amount of TH deposited into skin with Lamisil }{ }^{\circledR}
\end{gathered}
$$

Cumulative permeability was calculated as Equation (3):

$$
Q=\frac{C_{n} \times V_{0} \sum_{i=1}^{n=1} C_{i} \times V_{i}}{A}
$$

$C_{n}$ is the drug concentration measured at the nth sampling point, $V_{0}$ is the volume of the receiving medium in the receiving room, $C_{i}$ is the drug concentration measured at the $\mathrm{i}$-th sampling point, $V_{i}$ is the sampling volume, and $A$ is the effective transmission area of the diffusion cell. Steady-state transdermal flux $\left(J_{s s}\right)$ was calculated by taking the cumulative transmission $Q$ as the ordinate and the sampling time $t$ as the abscissa, and then linear regression is performed. The slope of the straight line is the steady-state transdermal rate $J_{s s}\left(\mu \mathrm{g} / \mathrm{cm}^{2} / \mathrm{h}\right)$.

\subsubsection{Visualization by Confocal Laser Scanning Microscopy (CLSM)}

To visualize the deposition of the formulations in different skin regions, a Confocal Laser Scanning Microscopy (CLSM) study was performed. Rhodamine B-labeled formulations (Rh B-ME-2, Rh B-BE, and Rh B-TE) were uniformly applied to intact skin of hairless SD rats at the concentration of $0.05 \%$ with amount of $1 \mathrm{~mL}$. After 1, 2, 4, and $8 \mathrm{~h}$ incubations, the marked area were excised with a surgical scalpel to remove subcutaneous tissues and fat and washed with distilled water [22]. A section of $\sim 20 \mu \mathrm{m}$ thickness was made by freezing microtome (Leica CM1950, Wetzlar, Germany). The photomicrographs of sections were observed by CLSM (Zeiss LSM880, Oberkochen, Germany) with the excitation and emission wavelengths at 488 and $532 \mathrm{~nm}$, respectively.

\subsection{Attenuated Total Reflection Fourier Transform Infrared Spectroscopy (ATR-FTIR)}

Rabbits were anesthetized with pentobarbital sodium (25\%,w/v, i.p.), and the back hair was removed by clippers, followed by drawing four squares $\left(1 \mathrm{~cm}^{2}\right)$. Then, $1 \mathrm{~mL}$ of ME-2, BE, and TE were applied evenly to the marked area. The rabbits were sacrificed after $60 \mathrm{~min}$ treatment to excise the treated skin. All the adipose tissues were removed for ATR-FTIR measurement. ATR-FTIR (Thermo Nicolet IS50, Madison, WI, USA) was equipped with diamond at an incident angle of $45^{\circ}$. Spectra were collected after 200 times scanning in the spectral range of 4000 to $1000 \mathrm{~cm}^{-1}$ [23]. Peak position was analyzed using Origin 2017 software. The skin treated with PBS was used as control.

\subsection{Skin Irritation Test and Histopathological Study}

The skin irritation tendency of the vesicles was evaluated by the Draize scoring method [24]. Rabbits were randomly divided into five groups of three animals each. The back of the animals was 
clipped free of hair. Then, $1 \mathrm{~mL}$ of the formulation was applied evenly to the hairless skin of the $1.25 \mathrm{~cm}^{2}$ area once daily for seven consecutive days. BE and ME-2 were applied to rabbits of group I and II, respectively. Commercially available TH cream was used for group III as control. Group IV was treated with saline as a negative control and group $\mathrm{V}$ with aqueous formaldehyde solution $(0.7 \%, v / v)$ as a positive control. Any signs of erythema and edema in the rabbit were observed for 7 days and scored on a scale of 0-4 (Erythema scale was evaluated as 0 , no erythema; 1 , slight; 2, well defined; 3 , moderate; 4 , scar formation; Edema scale was set as 0, none; 1 , slight; 2, well defined; 3 , moderate; 4 , severe).

After 7 days of skin irritation study, the rabbits were sacrificed. The application area was excised and fixed in $10 \%$ formalin for $12 \mathrm{~h}$. The embedded tissues in paraffin were cut into sections of $\sim 5 \mu \mathrm{m}$ thickness. The sections were stained with hematoxylin and observed using an optical microscope (Zeiss AXIO SCOPE A1, Oberkochen, Germany) for histopathological examination [25].

\subsection{In Vitro Antifungal Activity}

\subsubsection{Inoculum Preparation}

Candida albicans (C. albicans, ATCC 60193) was incubated on Sabouraud Dextrose Aga (SDA) for $24 \mathrm{~h}$ at $30^{\circ} \mathrm{C}$. Then, it was transferred to Sabouraud Dextrose broth medium and incubated overnight at $37^{\circ} \mathrm{C}$ with shaking (180 rpm) and diluted with sterile distilled water to $3 \times 10^{5} \mathrm{CFU} / \mathrm{mL}$, which was counted by hemocytometer [26].

\subsubsection{Minimum Inhibitory Concentration (MIC) Determination}

The MIC values against $C$. albicans were determined in 96-well microplates using a microdilution assay [27]. Briefly, the microdilution assay was performed by diluting three selected formulations (ME-2, BE, and TH-DMSO solution) with RPMI 1640 at concentrations of $1-0.125 \mu \mathrm{L} / \mathrm{mL}$. Then, $100 \mu \mathrm{L}$ of the diluted samples and C. albicans strain were mixed in wells of 96 microtiter plates (Thermo Scientific, Madison, WI, USA). The positive control group was performed using bacterial liquid and RPMI 1640 and the negative control group using RPMI 1640 . The plates were incubated for 4 days at $30^{\circ} \mathrm{C}, \mathrm{OD}_{600}$ (optical density) was determined with microplate reader, and the MIC was calculated.

\subsection{Statistical Analysis}

All the results in this study were expressed as mean \pm standard deviation (SD). Statistical analysis was accessed by one-way analysis of variance (ANOVA) and compared with Fisher's LSD post hoc test using SPSS 25.0 software (IBM Corporation, Armonk, NY, USA). $p<0.05$ was considered as statistically significant.

\section{Results and Discussion}

\subsection{Preparation and Characterization of the MEs}

TH-loaded MEs were successfully prepared with mean hydrodynamic diameter in the range of 100 to $120 \mathrm{~nm}$ (Table 1) with monodispersed size distribution (PDI <0.3). Interestingly, as shown in Table 1, an increasing tendency was observed for the mean hydrodynamic diamter from $103 \pm 0.14$ (ME-2) to $132 \pm 9.7 \mathrm{~nm}$ (BE) and PDI from $0.21 \pm 0.03$ (ME-3) to $0.30 \pm 0.05 \mathrm{~nm}$ (BE) when CA was removed in the system. This is the same case for TE formulation, where no PG was added, exhibited larger size $(124 \pm 6.9 \mathrm{~nm})$ and increased PDI (TE). In comparison, the CE vesicles without CA and PG exhibited the biggest mean hydrodynamic diameter $(152 \pm 7.2 \mathrm{~nm})$ with the largest PDI $(0.30 \pm 0.04)$. This indicates that the addition of CA and PG plays an important role in the particle size, including the mean hydrodynamic diameter and polydispersity. As shown in Figure 1, CA and PG could be located in the hydrophobic region of the lipid bilayer, contributing to reduced surface tension and increasing elasticity of the vesicles, thereby forming smaller particle sizes [28,29]. However, when the CA concentration further increased to $0.5 \%$, no significant differences were shown compared to 
$0.3 \%$ (100 \pm 8.9 and $103 \pm 9.3 \mathrm{~nm}$ for $0.3 \%$ and $0.5 \%$, respectively), indicating that $0.3 \%$ of CA is the best condition for this system. As a non-polar molecule, CA arranged mainly in the lipid bilayer. As the hydrophobic space was limited, lipid membrane could be saturated when more CA was added. In terms of particle size, the novel MEs are found smaller with more uniform particle size distribution. As illustrated in Figure 1, they displayed regular spherical morphology with fairly uniform particle size. The average particle size was $\sim 100 \mathrm{~nm}$, in perfect agreement with DLS analysis. The smaller size assists vesicles in penetrating across the $\mathrm{SC}$ to reach the targeting lesion.

The zeta of the nanoparticulate systems is an important factor that affects vesicle stability as well as interaction with the skin [30]. All the formulations prepared had a negative zeta ranging from -5.40 to $-13.02 \mathrm{mV}$ (Table 1). Repulsive forces between negatively charged vesicles contributed to improving the formulation stability. MEs systems showed similar zeta with TE formulations, but their absolute values were much higher than BE formulations, indicating better colloid stability.

Furthermore, the drug loading capacity was evaluated by calculating the drug EE. The EE reached up to $86 \% \pm 1.7 \%$ for the novel ME formulation (ME-2) despite of their smaller particle size. This is significantly higher than EE of the CE system $(60.9 \% \pm 1.9 \%)$, BE system $(65.9 \% \pm 1.9 \%)$, and TE system $(78.9 \% \pm 2.4 \%)$. The rigid strength of CE is limited by its drug loading capacity. The addition of PG could increase the TH solubilty, and, at the same time, it helped to reduce the volatilization of ethanol, making the outer membrane of the vesicles more stable. Moreover, PG was able to reduce the fluidity of lipids to ethanol, leading to decreased drug leakage from vesicles. Surpringly, the TE exhibited even higher EE then BE, which further improved by addition of PG.

In a nutshell, the optimized ME formulation (ME-2) was found to be the most promising TH delivery system considering the particle size, surface charge, and drug loading capacity. Therefore, ME-2 was selected to represent ME for all the following investigations.

\subsection{Stability Investigation}

Physicochemical stability studies were carried out at $4{ }^{\circ} \mathrm{C}$ and room temperature $\left(25^{\circ} \mathrm{C}\right)$ for 60 days. As presented in Figure 2, CE underwent a significant increase in particle size (from 152 to $169 \mathrm{~nm}$ ) and dramatic drug leakage (EE decreased from $60.9 \%$ to $53.5 \%$ ) during storage at $4{ }^{\circ} \mathrm{C}$. This phenomenon became more obvious when stored at $25^{\circ} \mathrm{C}$. BE vesicles could keep the drug payload at $4{ }^{\circ} \mathrm{C}$, due to the addition of PG. However, the colloid stability was not improved compared to the CE. On the contrary, TE exhibited great colloid stability at $4{ }^{\circ} \mathrm{C}$, but unfortunately drug migration was observed during storage at both 4 and $25^{\circ} \mathrm{C}$. Remarkabley, ME displayed both excellent colloid stability and no significant drug leakage was observed after 60 days storage at both 4 and $25^{\circ} \mathrm{C}$. These results indicate that the addition of both PG and CA significantly improve the colloid stability and prevent drug leakage.
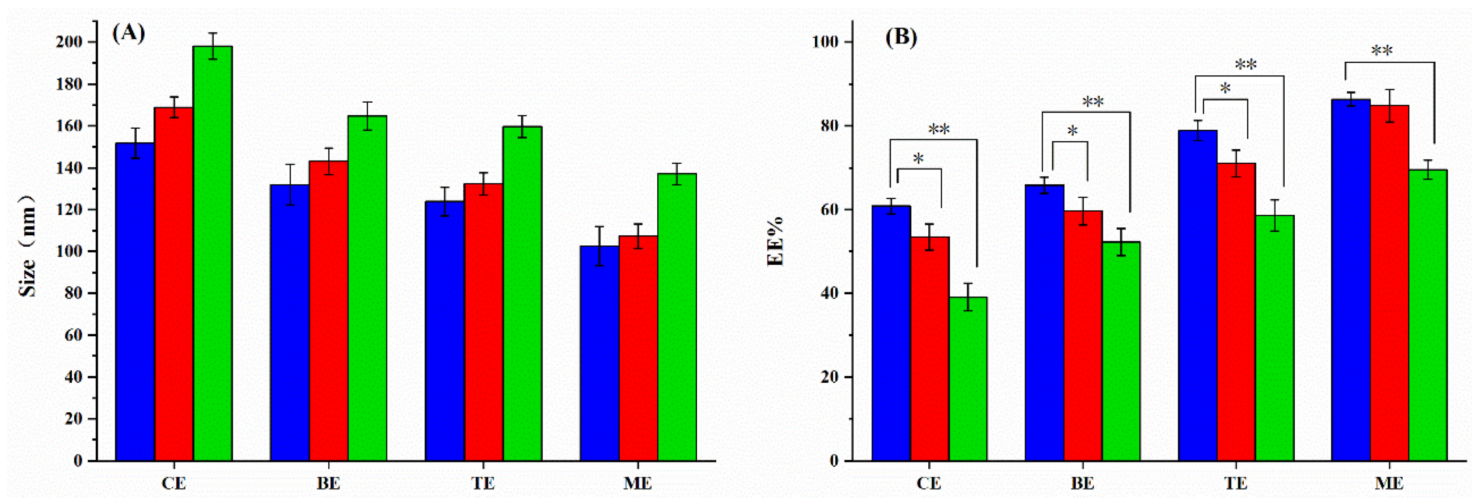

Figure 2. Stability investigation of the formulations after 60 days storage. (A) Particle size of different formulations before (blue) and after 60-day storage at 4 (red) or $25^{\circ} \mathrm{C}$ (green); (B) EE of different formulations before (blue) and after 60-day storage at 4 (red) or $25^{\circ} \mathrm{C}$ (green). Data are shown as the mean $\pm \mathrm{SD}(n=3)$. ${ }^{*} P<0.05,{ }^{* *} P<0.01$. 


\subsection{Skin Permeation and Deposition Studies}

To assess the effects of skin penetration and targeting properties of the selected formulations (ME, TE, BE, and CE), an ex vivo study was performed using a Franz diffusion cell method. The commercial cream Lamisil ${ }^{\circledR}$ was used as control. Steady-state transdermal flux $\left(J_{s s}\right)$ was calculated to directly compare the permeation rate differences across the formulations. It was found that $J_{S S}$ of Lamisil ${ }^{\circledR}$ cream was $0.587\left(\mu \mathrm{g} \cdot \mathrm{cm}^{-1} \cdot \mathrm{h}^{-1}\right)$; however, the $J_{s s}$ values of all the tested ethosomes $(\mathrm{CE}, \mathrm{BE}$, $\mathrm{TE}$, and ME) was too low to be detected. The cumulative permeability in $24 \mathrm{~h}\left(Q_{24 \mathrm{~h}}\right)$ of the Lamisil ${ }^{\circledR}$ cream was found as $6.47 \pm 1.02 \mu \mathrm{g} \cdot \mathrm{cm}^{-2}$; by contrast, the $Q_{24 \mathrm{~h}}$ of all the ethosome-based formulations was below the determination limit $(50 \mathrm{ng} / \mathrm{mL}$ ). It can be clearly seen from the results that ethosome ( $\mathrm{CE}, \mathrm{BE}, \mathrm{TE}$, and $\mathrm{ME}$ )-based vesicles help to remain the drug in the deep skin, but the drug cannot easily penetrate into the receiving pool.

As shown in Figure 3, the TH amount in both the SC and epidermis/dermis layer increased with longer deposition time. The maximal value was reached at $24 \mathrm{~h}$. In the case of Lamisil ${ }^{\circledR}$, the TH amount in the SC and epidermis/dermis layer after $24 \mathrm{~h}$ deposition reached $9.9 \% \pm 0.9 \%$ and $5.6 \% \pm 1.1 \%$, respectively. This indicates that the cream Lamisil ${ }^{\circledR}$ poorly penetrated across SC, with only $5.6 \% \pm 1.1 \%$ of $\mathrm{TH}$ reach the deeper skin. Compared with Lamisil ${ }^{\circledR}$, all the ethosomes formulations had significant higher TH content in epidermis/dermis layer, suggesting much stronger penetrating capacity of ethosomes formulations. In the case of $\mathrm{CE}$, the $\mathrm{TH}$ amount in epidermis/dermis layer increased to $8.8 \% \pm 1.2 \%$. This value was further improved by addition of PG $(12.3 \% \pm 1.2 \%$ for $\mathrm{BE})$ or CA $(10.1 \% \pm 1.3 \%$ for TE). Remarkably, when the ME was applied, a much higher deeper skin deposition amount reached up to $18.2 \% \pm 1.3 \%$, which was 3.3 times higher than that of the commercially available cream. This clearly shows the synergistic effect of the addition of both PG and CA in the vesicles.
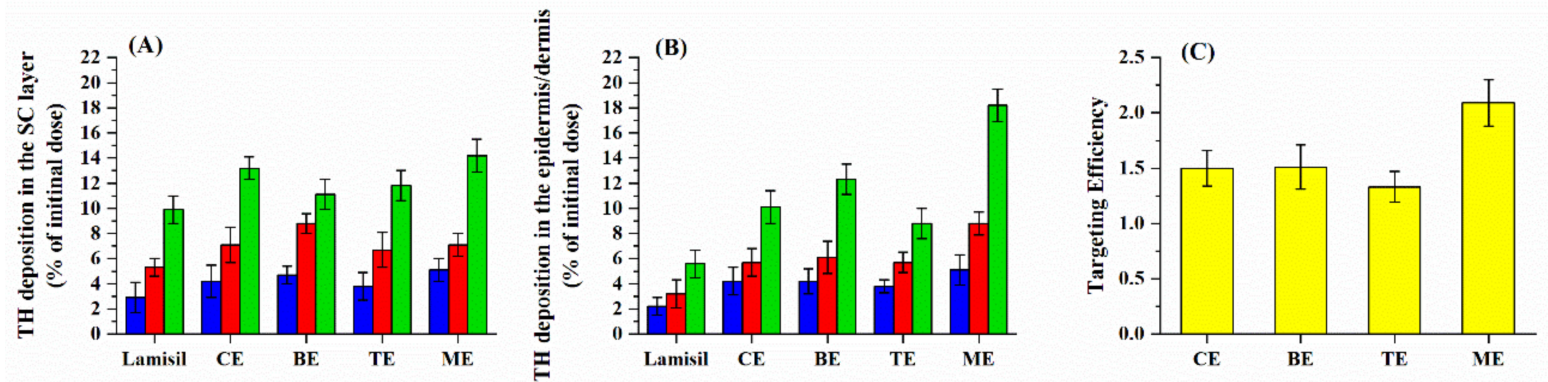

Figure 3. The amount of TH in different layers of rat skin after deposition at $6 \mathrm{~h}$ (blue), $12 \mathrm{~h}$ (red), and $24 \mathrm{~h}$ (green) $(n=6)$. (A) TH deposition in the SC layer; (B) TH deposition in the epidermis/dermis; (C) Targeting efficiency of different formulations. Data are shown as the mean $\pm \operatorname{SD}(n=6)$.

Targeting efficiency is a parameter used to assess skin targeting properties of topical formulations [31]. The results in Figure 3C showed the targeting efficiency of the selected ethosomes vesicles after $24 \mathrm{~h}$ deposition. It was found that $\mathrm{CE}$ possessed a targeting efficiency value of 1.32 , which was further improved by BE (1.51) or TE (1.50). However, the highest targeting efficiency up to 2.09 was obtained with ME. It clearly indicates that ME can efficiently promote drug penetration and target to the epidermal dermis, forming a drug reservoir to release the drug. This is a very promising strategy to improve the drug efficiency and therefore decrease the risk of side effects.

The ethosomal formulation can fluidize the skin lipid membrane and accordingly enhance drug deposition [32]. Ethanol and propylene glycol can reduce the phase transition temperature of SC [33]. At the same time, the ethosomal formulation acts as a deformable vesicle, and the added Tween- 80 acts as an edge activator, which increases its deformability and easily penetrates the gaps of the SC to reach deeper skin. When phospholipids interact with SC, the drug is released to form a micro-drug reservoir [34]. 


\subsection{Tracking the Fluorescently-Labeled Vesicles in the Rat Skin Layers Using CLSM}

The selected ME as well as CE and BE were loaded with a fluorescent dye (Rh B), instead of the drug. This enables the tracking of vesicles and their distributions in different skin layers. All experimental conditions (including the concentration of the vesicles and the dose of the fluorescent dye in vesicles) were kept constant for precise comparison.

The distribution of Rh B-loaded vesicles within the rat skin layers after different application time are represented in Figure 4. It is observed that the fluorescence intensity appeared mainly in the SC layer associated with a discrete localization in the epidermis and the dermis, for all formulations within the first $2 \mathrm{~h}$ of application. As the duration of action increases, the fluorescence intensity also increases, indicating that vesicle penetration was a time-dependent process. In case of $\mathrm{CE}$ and $\mathrm{BE}$, $\mathrm{Rh} \mathrm{B}$ accumulated onto the skin surface (e.g., SC) in $4 \mathrm{~h}$, thus indicating the limited penetration to deeper layers. Interestingly, it is clearly seen that, when treated with ME, the fluorescence intensity in the deeper skin was higher than that of the control (CE and BE) and the red band was much more pronounced. It suggestes that the addition of CA significantly improved the penetration across SC into the epidermis and the dermis layer. After $8 \mathrm{~h}$ of treatment, significant fluorescence intensity is observed in the epidermis and the dermis when treated with $\mathrm{CE}$, however this signal was very weak. In contrast, much higher signal was observed when treated with BE, reflecting the enhanced permeation. This is possibly because of the high elasticity and flexibility of BE, attributed to the presence of PG. Moreover, it is illustrated that the hair follicles had a higher fluorescence intensity than other area, proving that some drugs/ethosomes enter the skin possibly through the skin appendage pathway. As expected, the highest fluorescence intensity was found when treated with ME, where both PG and CA were present, further confirming the necessity of the added CA. These resultes are consistent with the ex vivo permeation experiment and further confirm the targeting effect of ME.

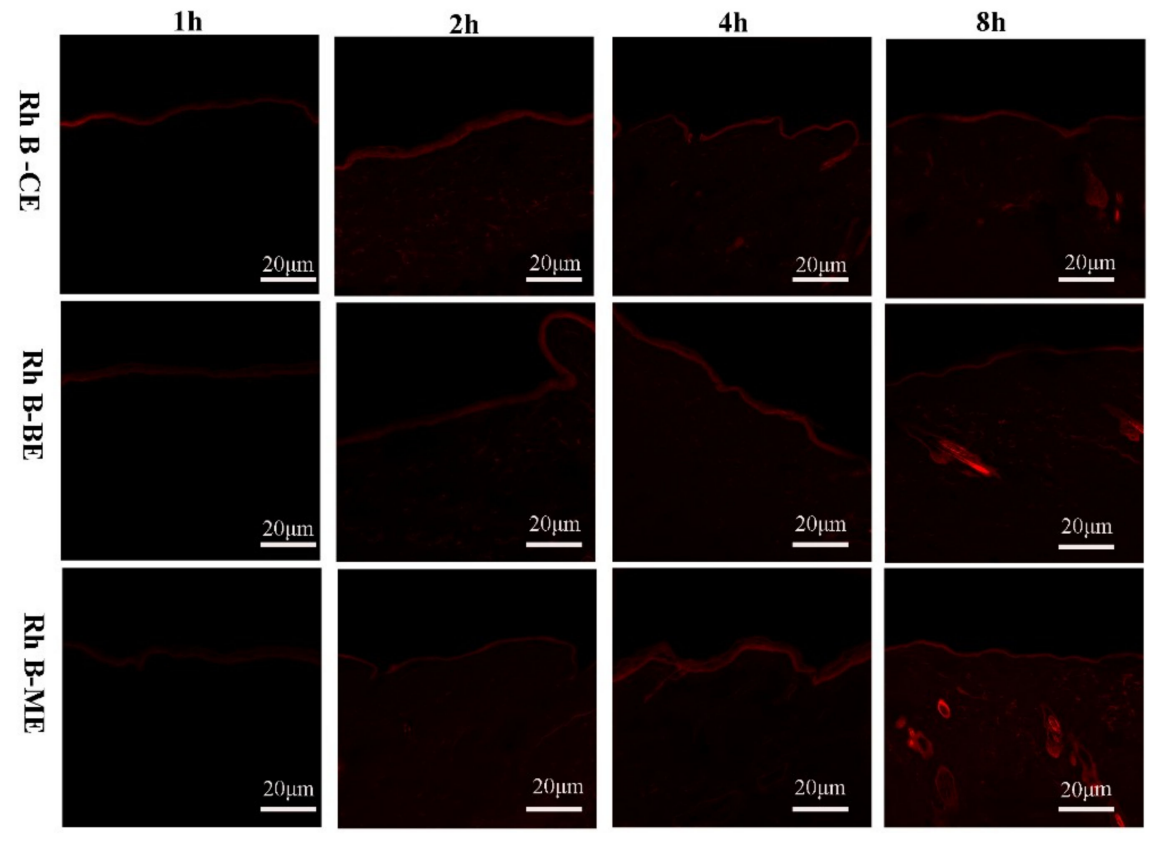

Figure 4. Confocal Laser Scanning Microscopy (CLSM) images of vertical section of rat skin incubated with Rh B labeled formulations at 1, 2, 4, and $8 \mathrm{~h}$ post-treatment.

\subsection{ATR-FTIR}

ATR-FTIR is a powerful non-destructive technique used to explore the mechanisms of ethosome transdermal permeation, by characterizing the SC spectral information at the molecular level. The main absorption peaks of the skin were shown in Figure 5A. The peaks between 3000 and $3600 \mathrm{~cm}^{-1} \mathrm{are}^{-}$ attributed to $\mathrm{O}-\mathrm{H}$ and $\mathrm{N}-\mathrm{H}$ stretches from protein, water, and lipid in the skin. In particular, $\mathrm{CH}_{2}$ 
asymmetric $\left(\sim 2921 \mathrm{~cm}^{-1}\right)$ and $\mathrm{CH}_{2}$ symmetric stretching vibration $\left(\sim 2853 \mathrm{~cm}^{-1}\right)$ correspond to SC intercellular lipids [35]. It is of great interest to study the $\mathrm{CH}_{2}$ stretching vibration, which indicates a change in the SC lipids. Analyses of these bands allows further understanding of the change in the skin structure. After the treatment with different ethosomes, the wavenumber of $\mathrm{CH}_{2}$ symmetric and asymmetric stretching were shown in Figure 5B,C. A significant blue shift (higher wavenumber value) was observed for ME group compared with the control samples (2853.82 and $2853.13 \mathrm{~cm}^{-1}$ for ME group and control, repectively), indicating a lipid order-disorder transition. This transition also contributes to an increase of the SC lipids fluidity, which potentially increase the skin penetration of TH [36]. Moreover, after treatment with $\mathrm{ME}$, the absorption intensity of the $\mathrm{C}-\mathrm{H}$ symmetric and asymmetric stretching vibration was significantly weakened. However, no significant differences were found for the groups treated with TE, BE, and PBS (control), indicating that both PG and CA are required for this formulation to affect the structure of the $\mathrm{SC}$ layer.

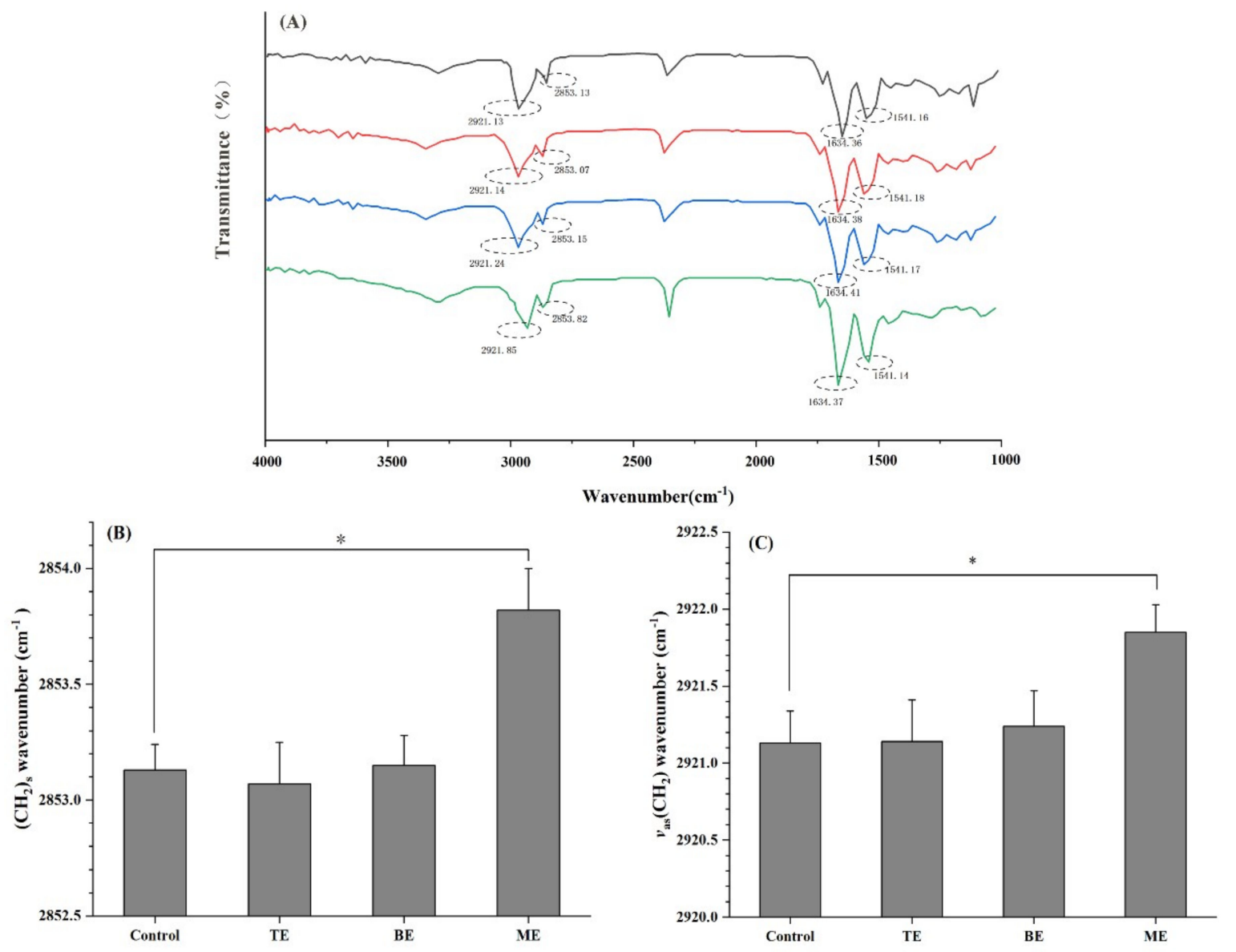

Figure 5. ATR-FTIR investigation. (A) ATR-FTIR transmission spectra of rabbit skin treated with TE (red), BE (blue), ME (green), and saline (black, as control). (B,C) Wavenumbers of symmetric and asymmetric methylene stretching of SC lipids. Mean $\pm \mathrm{SD}, n=4$; ${ }^{*}$ Statistically significant difference compared to the respective control at $p<0.05$.

The peaks at 1634 and $1541 \mathrm{~cm}^{-1}$ are related to the $\mathrm{C}-\mathrm{O}$ stretching (amide I) stretching and $\mathrm{C}-\mathrm{N}$ stretching (amide II) linkage of the helical secondary structure in epidermal keratin, respectively. No significant differences were observed for amide I and amide II bands with and without the ethosome treatment (Figure S2), indicating that the keratin in SC was not hydrolyzed and the integrity of SC was maintained [37]. 
Therefore, the ATR-FTIR spectra reveal that after ME treatment the lipid fluidity of the SC barrier was increased, which is favorable to enhance the transdermal permeation of $\mathrm{TH}$, but the integrity of the SC was not altered.

\subsection{Skin Irritation Study and Histological Observation}

Skin irritation and toxicity assay of a topical formulation is crucial because of the potential adverse effects [37]. In this study, erythema and edema scores were evaluated to assess their skin irritation. As shown in Table 2, the scores of different formulations were listed. Formalin $(0.8 \%)$ was used as positive control, showing erythema and edema scores of $2.75 \pm 0.50$ and $2.25 \pm 0.57$, respectively. PBS was used as negative control. The commercial Lamisil ${ }^{\circledR}$ exhibited slightly skin irradiation with erythema and edema scores of $1.75 \pm 0.50$ and $1.25 \pm 0.50$, respectively. Remarkably, both BE and ME formulations displayed much less erythema and edema (all the scores $\leq 0.75$ ), indicating non-irritating property.

To evaluate the biocompatibility of the ME formulation to the skin, we performed the histopathological characterization of the skin after treated with ME formulations. The commercial cream Lamisil ${ }^{\mathbb{R}}$ was evaluated in the same manner. As shown in Figure 6A, when the skin was treated with PBS, there was a complete SC layer located at the topmost layer of the epidermis, and the boundary between the epidermis and the dermis was clear. No infiltration of inflammatory cells were observed in the dermis, and the skin appendages were normal. After administration with BE and ME vesicles, there was no significant difference in SC integrity as compared to the control (Figure 6). No evidence for the presence of inflammatory cells was found. The phospholipid in BE and ME is a lipophilic component, which is not destructive to the skin. Owing to the good elasticity of ME and the penetration enhancer (CA) it contains, it can efficiently penetrate cross the skin barrier, targeting to the demis area. However, it did not damage the integrity of the SC.
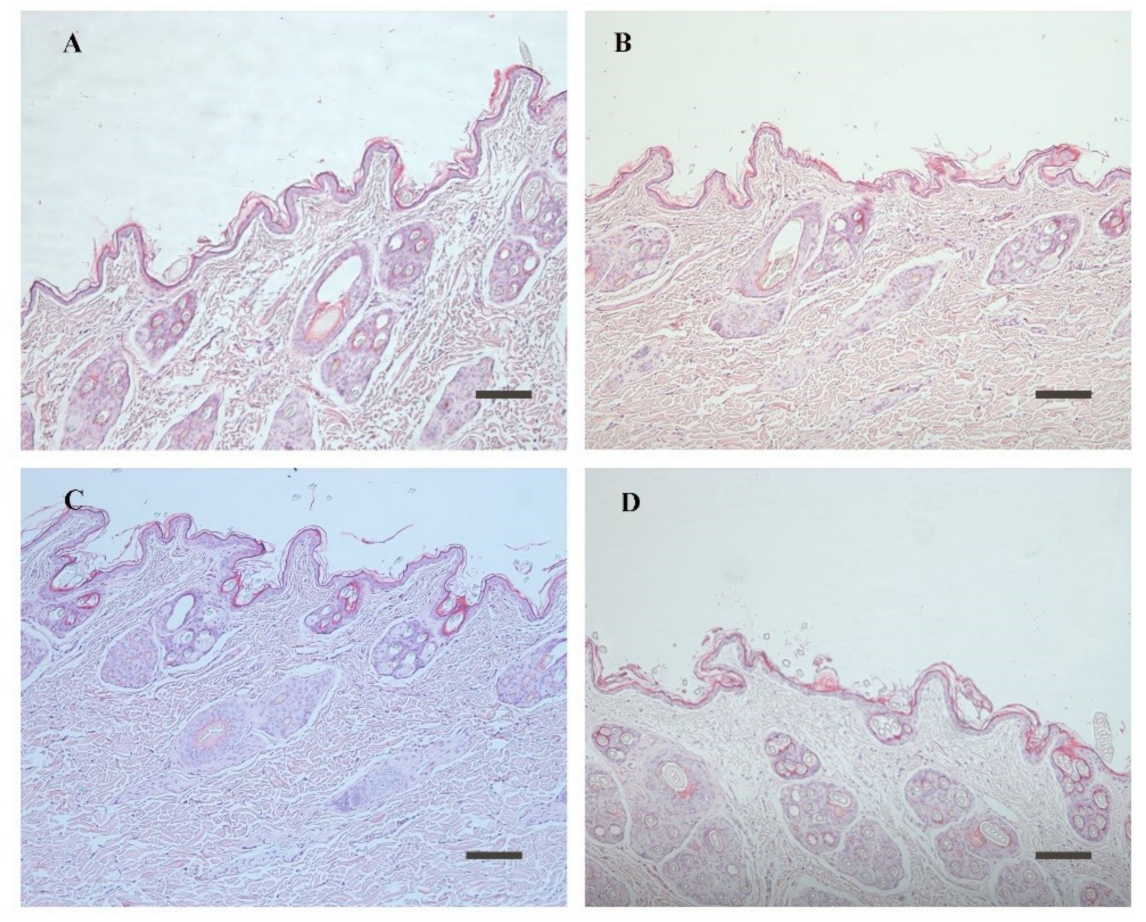

Figure 6. Histological micrograph of rat skin after treatment with: (A) BE; (B) ME; (C) Lamisil ${ }^{\circledR}$ cream; and (D) PBS. (The upper layer in the picture represents SC. Scale bar: $200 \mu \mathrm{m}$.). 
Table 2. Score for skin irritation study on rabbit calculated after 7 days.

\begin{tabular}{ccc}
\hline & Groups & Score \pm SD \\
\hline \multirow{2}{*}{ BE (group I) } & Erythema & $0.50 \pm 0.57$ \\
& Edema & $0.50 \pm 0.57$ \\
ME (group II) & Erythema & $0.75 \pm 0.50$ \\
& Edema & $0.50 \pm 0.57$ \\
Lamisil ${ }^{\circledR}$ (groupIII) & Erythema & $1.75 \pm 0.50$ \\
PBS (group IV) & Edema & $1.25 \pm 0.50$ \\
\multirow{2}{*}{ Formaline (group V) } & Erythema & $0.00 \pm 0.00$ \\
& Edema & $0.00 \pm 0.00$ \\
& Erythema & $2.75 \pm 0.50$ \\
& Edema & $2.25 \pm 0.50$ \\
\hline
\end{tabular}

\subsection{Antifungal Activity}

The antifungal activity of BE and ME was tested on C. albicans strains by the determination of minimum inhibitory concentration (MIC). TH dissolved in DMSO was used as control. The results reveal that the $\mathrm{TH}$ has antifungal activity with $\mathrm{MIC}_{50}$ of $0.25 \mu \mathrm{g} / \mathrm{mL}$ (Table 3). This activity was significantly improved when TH was loaded in BE ( $\mathrm{MIC}_{50}$ of $0.0625 \mu \mathrm{g} / \mathrm{mL}, p<0.05$ for TH-DMSO and $\mathrm{BE}$ ). This is possibly due to the better interaction of the vesicles (phospholipid) with the fungal membrane. Remarkably, with the addition of CA, ME displayed significantly smaller $\mathrm{MIC}_{50}$ in comparison with $\mathrm{BE}$, indicating that $\mathrm{CA}$ could efficiently enhance the $\mathrm{TH}$ penetration cross the fungal membrane $(p<0.05$ for $\mathrm{BE}$ and $\mathrm{ME})$.

Table 3. In vitro antifungal activity of TH, TH-loaded BE, and ME on C. albicans determined by MIC.

\begin{tabular}{cccc}
\hline Antifungal Agent & MIC Range, $\mu \mathrm{g} / \mathrm{mL}$ & MIC $_{\mathbf{5 0}}(\boldsymbol{\mu g} / \mathrm{mL})$ & MIC $_{\mathbf{9 0}}(\boldsymbol{\mu g} / \mathrm{mL})$ \\
\hline TH-DMSO & $0.125-1$ & 0.25 & 1 \\
BE & $0.0312-0.25$ & $0.0625^{*}$ & $0.25^{*}$ \\
ME & $0.0156-0.125$ & $0.0313^{* *}$ & $0.0625^{* *}$ \\
\hline
\end{tabular}

Note: $\mathrm{MIC}_{50}, \mathrm{MIC}_{90}: 50 \%$ and $90 \%$ of fungi are inhibited respectively. ${ }^{*} P<0.05,{ }^{* *} P<0.01$.

These results clearly indicated that BE containing CA could significantly improve the in vitro antifungal effect on C. albicans. In other words, lower drug doses will be required for C. albicans treatment when $\mathrm{TH}$ is encapsulated in ME, which could improve patient compliance, decrease the incidence and severity of side effects, and lead to better therapeutic outcome.

Compared to the literature reporting on TH containing nanoparticles, especially liposomes [38], ethosomes [39], sonicated ethosomes [40], binary ethosomes [6], and transfersomes [41], the main advantage of the novel MS is that for the first time a functional (antifungal) element CA was added in the system, which helps both the formation of the nanovesicles and, at the same time, synergistically contributes to the antifungal activity.

\section{Conclusions}

In this study, TH-loaded MEs with homogeneous particle sizes of $\sim 100 \mathrm{~nm}$ were successfully prepared via a cold method. High TH entrapment efficiency reached up to $86 \%$. By addition of both PG and CA, MEs possessed excellent colloid stability with no drug leakage during 2 months storage. Ex vivo permeability and deposition studies showed that ME exhibited the targeting property, as compared to a commercial Lamisil ${ }^{\circledR}$ cream as well as the prepared BE and TE. Confocal microscopic investigation further confirms this targeting effect by visualizing how Rhodamine-labeled MEs penetrate across SC into the epidermis and the dermis layer. Moreover, ME was found biocompatible, confirmed by irritation and allergy tests. Finally, the antibacterial activity of ME was tested on C. albicans strains by the determination of MIC, showing significantly reduced MIC values in contrast to TH drug 
solution and BE formulations. This study paves the way towards design of dermal targeting ME for fungal/bacterial infection treatment.

Supplementary Materials: The following are available online at http://www.mdpi.com/2079-6412/10/4/304/s1, Figure S1: The TH solubility in different mediums at $37 \pm 0.5^{\circ} \mathrm{C}$. Data are shown as the mean $\pm \mathrm{SD}(n=3)$, Figure S2: ATR-FTIR investigation: Amide I (A) and amide II (B) wavenumbers of the rabbit skin treated with different formulations.

Author Contributions: Conceptualization, L.Z.; Methodology, S.S., L.Z. and X.L.; Validation, M.D., L.Z. and S.Z.; Formal Analysis, X.L. and S.Z.; Investigation, L.Z., A.M. and T.Z.; Writing-Original Draft Preparation, L.Z.; Writing-Review and Editing, L.Z., X.L. and A.M.; Supervision, X.L.; Project Administration, S.S. and L.Z.; Funding Acquisition, T.Z. and M.D. All authors have read and agreed to the published version of the manuscript.

Funding: This research was funded by the National Natural Science Foundation of China (No. 81903808) and the Natural Science Foundation of Zhejiang Province (Grant No. LGC19H280003).

Acknowledgments: The present work has benefited from the Experimentation Animal Service of Zhejiang Chinese Medical University, the Academy of Traditional Chinese Medicine of Zhejiang Chinese Medical University.

Conflicts of Interest: The authors declare no conflicts of interest.

\section{References}

1. Chowdhary, A.; Prakash, A.; Sharma, C.; Kordalewska, M.; Kumar, A.; Sarma, S.; Tarai, B.; Singh, A.; Upadhyaya, G.; Upadhyay, S.; et al. A multicentre study of antifungal susceptibility patterns among 350 Candida auris isolates (2009-17) in India: Role of the ERG11 and FKS1 genes in azole and echinocandin resistance. J. Antimicrob. Chemother. 2018, 73, 891-899. [CrossRef] [PubMed]

2. Rosen, T.; Gold, L.F.S. Antifungal drugs for onychomycosis: Efficacy, safety, and mechanisms of action. Semin. Cutan. Med. Surg. 2016, 35, S51-S55. [CrossRef] [PubMed]

3. Lusiana Muller-Goymann, C.C. Preparation, characterization, and in vitro permeation study of terbinafine $\mathrm{HCl}$ in poloxamer 407-based thermogelling formulation for topical application. AAPS PharmSciTech 2011, 12, 496-506. [CrossRef] [PubMed]

4. Nozawa, M.; Goto, M.; Wada, Y.; Kumazawa, M.; Shimokawa, K.-I.; Ishii, F. Generic selection criteria for safety and patient benefit VII: Comparing the physicochemical and pharmaceutical properties of brand-name and generic terbinafine hydrochloride cream. Drug Discov. Ther. 2018, 12, 16-20. [CrossRef]

5. Ashtikar, M.; Nagarsekar, K.; Fahr, A. Transdermal delivery from liposomal formulations-Evolution of the technology over the last three decades. J. Control. Release 2016, 242, 126-140. [CrossRef]

6. Zhang, J.P.; Wei, Y.H.; Zhou, Y.; Li, Y.Q.; Wu, X.A. Ethosomes, binary ethosomes and transfersomes of terbinafine hydrochloride: A comparative study. Arch. Pharm. Res. 2012, 35, 109-117. [CrossRef]

7. Moolakkadath, T.; Aqil, M.; Ahad, A.; Imam, S.S.; Praveen, A.; Sultana, Y.; Mujeeb, M.; Iqbal, Z. Fisetin loaded binary ethosomes for management of skin cancer by dermal application on UV exposed mice. Int. J. Pharm. 2019, 560, 78-91. [CrossRef]

8. Song, C.K.; Balakrishnan, P.; Shim, C.K.; Chung, S.J.; Chong, S.; Kim, D.D. A novel vesicular carrier, transethosome, for enhanced skin delivery of voriconazole: Characterization and in vitro/in vivo evaluation. Colloid Surf. B-Biointerfaces 2012, 92, 299-304. [CrossRef]

9. Zhou, Y.; Wei, Y.H.; Liu, H.X.; Zhang, G.Q.; Wu, X.A. Preparation and in vitro evaluation of ethosomal total alkaloids of sophora alopecuroides loaded by a transmembrane $\mathrm{pH}$-gradient method. AAPS PharmSciTech 2010, 11, 1350-1358. [CrossRef]

10. Sala, M.; Diab, R.; Elaissari, A.; Fessi, H. Lipid nanocarriers as skin drug delivery systems: Properties, mechanisms of skin interactions and medical applications. Int. J. Pharm. 2018, 535, 1-17. [CrossRef]

11. Abdel-Messih, H.A.; Ishak, R.A.H.; Geneidi, A.S.; Mansour, S. Tailoring novel soft nano-vesicles 'Flexosomes' for enhanced transdermal drug delivery: Optimization, characterization and comprehensive ex vivo-In vivo evaluation. Int. J. Pharm. 2019, 560, 101-115. [CrossRef] [PubMed]

12. Chen, L.; Wang, Z.; Liu, L.; Qu, S.; Mao, Y.Y.; Peng, X.; Li, Y.X.; Tian, J. Cinnamaldehyde inhibits Candida albicans growth by causing apoptosis and its treatment on vulvovaginal candidiasis and oropharyngeal candidiasis. Appl. Microbiol. Biotechnol. 2019, 103, 9037-9055. [CrossRef] [PubMed] 
13. Feng, X.; Chen, Y.T.; Li, L.Y.; Zhang, Y.Q.; Zhang, L.T.; Zhang, Z.Q. Preparation, evaluation and metabolites study in rats of novel amentoflavone-loaded TPGS/soluplus mixed nanomicelles. Drug Deliv. 2020, 27, 137-150. [CrossRef] [PubMed]

14. Sharma, G.; Goyal, H.; Thakur, K.; Raza, K.; Katare, O.P. Novel elastic membrane vesicles (EMVs) and ethosomes-mediated effective topical delivery of aceclofenac: A new therapeutic approach for pain and inflammation. Drug Deliv. 2016, 23, 3135-3145. [CrossRef] [PubMed]

15. El-Kayal, M.; Nasr, M.; Elkheshen, S.; Mortada, N. Colloidal (-)-epigallocatechin-3-gallate vesicular systems for prevention and treatment of skin cancer: A comprehensive experimental study with preclinical investigation. Eur. J. Pharm. Sci. 2019, 137, 15. [CrossRef] [PubMed]

16. Kassem, A.A.; Mohsen, A.M.; Ahmed, R.S.; Essam, T.M. Self-nanoemulsifying drug delivery system (SNEDDS) with enhanced solubilization of nystatin for treatment of oral candidiasis: Design, optimization, in vitro and in vivo evaluation. J. Mol. Liq. 2016, 218, 219-232. [CrossRef]

17. Ma, M.; Wang, J.P.; Guo, F.; Lei, M.Z.; Tan, F.P.; Li, N. Development of nanovesicular systems for dermal imiquimod delivery: Physicochemical characterization and in vitro/in vivo evaluation. J. Mater. Sci. Mater. Med. 2015, 26, 1-11. [CrossRef]

18. Chu, Y.; Li, D.; Luo, Y.F.; He, X.J.; Jiang, M.Y. Preparation and in vitro evaluation of glycyrrhetinic acid-modified curcumin-loaded nanostructured lipid carriers. Molecules 2014, 19, 2445-2457. [CrossRef]

19. Ahmed, O.A.A.; Badr-Eldin, S.M. Development of an optimized avanafil-loaded invasomal transdermal film: Ex vivo skin permeation and in vivo evaluation. Int. J. Pharm. 2019, 570, 118657. [CrossRef]

20. Lademann, J.; Ilgevicius, A.; Zurbau, O.; Liess, H.D.; Schanzer, S.; Weigmann, H.J.; Antoniou, C.; Pelchrzim, R.V.; Sterry, W. Penetration studies of topically applied substances: Optical determination of the amount of stratum corneum removed by tape stripping. J. Biomed. Opt. 2006, 11, 054026. [CrossRef]

21. Marttin, E.; Neelissen-Subnel, M.T.; De Haan, F.H.; Bodde, H.E. A critical comparison of methods to quantify stratum corneum removed by tape stripping. Skin Pharm. Off. J. Skin Pharmacol. Soc. 1996, 9, 69-77. [CrossRef] [PubMed]

22. Godin, B.; Touitou, E. Mechanism of bacitracin permeation enhancement through the skin and cellular membranes from an ethosomal carrier. J. Control. Release Off. J. Control. Release Soc. 2004, 94, 365-379. [CrossRef] [PubMed]

23. Kazarian, S.G.; Chan, K.L.A. Applications of ATR-FTIR spectroscopic imaging to biomedical samples. Biochim. Biophys. Acta 2006, 1758, 858-867. [CrossRef] [PubMed]

24. Morales, M.; Perez, D.; Correa, L.; Restrepo, L. Evaluation of fibrin-based dermal-epidermal organotypic cultures for in vitro skin corrosion and irritation testing of chemicals according to OECD TG 431 and 439. Toxicol. In Vitro 2016, 36, 89-96. [CrossRef]

25. Negi, P.; Singh, B.; Sharma, G.; Beg, S.; Raza, K.; Katare, O.P. Phospholipid microemulsion-based hydrogel for enhanced topical delivery of lidocaine and prilocaine: QbD-based development and evaluation. Drug Deliv. 2016, 23, 951-967. [CrossRef]

26. Fekrazad, R.; Barghi, V.G.; Mir, A.P.B.; Shams-Ghahfarokhi, M. In vitro photodynamic inactivation of Candida albicans by phenothiazine dye (new methylene blue) and lndocyanine green (EmunDo (R)). Photodiagnosis Photodyn. Ther. 2015, 12, 52-57. [CrossRef]

27. Elshikh, M.; Ahmed, S.; Funston, S.; Dunlop, P.; McGaw, M.; Marchant, R.; Banat, I.M. Resazurin-based 96-well plate microdilution method for the determination of minimum inhibitory concentration of biosurfactants. Biotechnol. Lett. 2016, 38, 1015-1019. [CrossRef]

28. Bilbao-Sainz, C.; Chiou, B.-S.; Du, W.-X.; Gregorsky, K.S.; Orts, W.J. Influence of disperse phase characteristics on stability, physical and antimicrobial properties of emulsions containing cinnamaldehyde. J. Am. Oil Chem. Soc. 2013, 90, 233-241. [CrossRef]

29. Nainwal, N.; Jawla, S.; Singh, R.; Saharan, V.A. Transdermal applications of ethosomes-A detailed review. J. Liposome Res. 2019, 29, 103-113. [CrossRef]

30. Wang, J.; Ma, C.; Guo, C.; Yuan, R.; Zhan, X. CTG-loaded liposomes as an approach for improving the intestinal absorption of asiaticoside in Centella Total Glucosides. Int. J. Pharm. 2016, 509, 296-304. [CrossRef]

31. Liu, J.; Hu, W.; Chen, H.; Ni, Q.; Xu, H.; Yang, X. Isotretinoin-loaded solid lipid nanoparticles with skin targeting for topical delivery. Int. J. Pharm. 2007, 328, 191-195. [CrossRef] [PubMed]

32. Dayan, N.; Touitou, E. Carrrers for skin delivery of trihexyphenidyl HCl: Ethosomes vs. liposmes. Biomaterials 2000, 21, 1879-1885. [CrossRef] 
33. Verma, P.; Pathak, K. Nanosized ethanolic vesicles loaded with econazole nitrate for the treatment of deep fungal infections through topical gel formulation. Nanomed. Nanotechnol. Biol. Med. 2012, 8, 489-496. [CrossRef]

34. Verma, D.D.; Verma, S.; Blume, G.; Fahr, A. Particle size of liposomes influences dermal delivery of substance into skin. Int. J. Pharm. 2003, 258, 141-151. [CrossRef]

35. Obata, Y.; Utsumi, S.; Watanabe, H.; Suda, M.; Tokudome, Y.; Otsuka, M.; Takayama, K. Infrared spectroscopic study of lipid interaction in stratum corneum treated with transdermal absorption enhancers. Int. J. Pharm. 2010, 389, 18-23. [CrossRef] [PubMed]

36. Wang, H.; Tian, Q.; Quan, P.; Liu, C.; Fang, L. Probing the role of ion-pair strategy in controlling dexmedetomidine penetrate through drug-in-adhesive patch: Mechanistic insights based on release and percutaneous absorption process. AAPS PharmSciTech 2020, 21, 1-14. [CrossRef]

37. Hertzberg, O.; Bauer, A.; Kuederle, A.; Pleitez, M.A.; Maentele, W. Depth-selective photothermal IR spectroscopy of skin: Potential application for non-invasive glucose measurement. Analyst 2017, 142, 495-502. [CrossRef]

38. Yang, Y.; Ou, R.J.; Guan, S.X.; Ye, X.L.; Hu, B.; Zhang, Y.; Lu, S.F.; Zhou, Y.B.; Yuan, Z.W.; Zhang, J.; et al. A novel drug delivery gel of terbinafine hydrochloride with high penetration for external use. Drug Deliv. 2015, 22, 1086-1093. [CrossRef]

39. Iizhar, S.A.; Syed, I.A.; Satar, R.; Ansari, S.A. In vitro assessment of pharmaceutical potential of ethosomes entrapped with terbinafine hydrochloride. J. Adv. Res. 2016, 7, 453-461. [CrossRef]

40. Abdulbaqi, I.M.; Darwis, Y.; Khan, N.A.K.; Abou Assi, R.; Khan, A.A. Ethosomal nanocarriers: The impact of constituents and formulation techniques on ethosomal properties, in vivo studies, and clinical trials. Int. J. Nanomed. 2016, 11, 26. [CrossRef]

41. Koutsoulas, C.; Pippa, N.; Demetzos, C.; Zabka, M. Preparation of liposomal nanoparticles incorporating terbinafine in vitro drug release studies. J. Nanosci. Nanotechnol. 2014, 14, 4529-4533. [CrossRef] [PubMed]

(C) 2020 by the authors. Licensee MDPI, Basel, Switzerland. This article is an open access article distributed under the terms and conditions of the Creative Commons Attribution (CC BY) license (http://creativecommons.org/licenses/by/4.0/). 